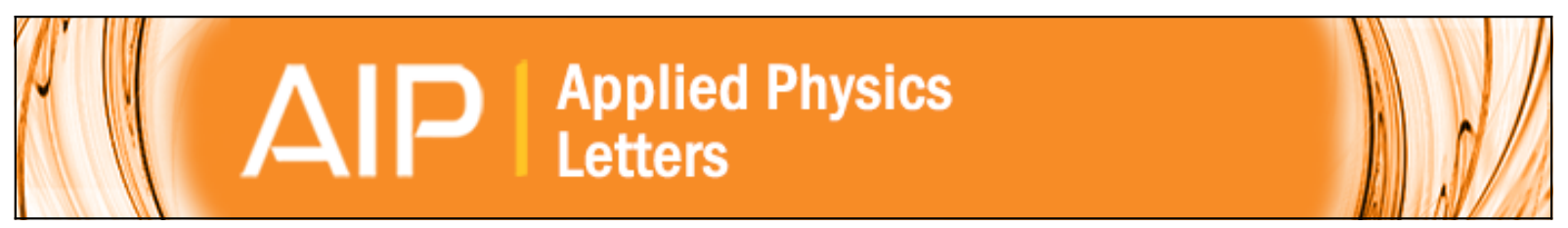

\title{
Time-resolved scanning electron microscopy with polarization analysis
}

Robert Frömter, Fabian Kloodt, Stefan Rößler, Axel Frauen, Philipp Staeck, Demetrio R. Cavicchia, Lars

Bocklage, Volker Röbisch, Eckhard Quandt, and Hans Peter Oepen

Citation: Applied Physics Letters 108, 142401 (2016); doi: 10.1063/1.4945053

View online: http://dx.doi.org/10.1063/1.4945053

View Table of Contents: http://scitation.aip.org/content/aip/journal/apl/108/14?ver=pdfcov

Published by the AIP Publishing

\section{Articles you may be interested in}

A compact electron gun for time-resolved electron diffraction

Rev. Sci. Instrum. 86, 013109 (2015); 10.1063/1.4905335

Scanning photoelectron microscope for nanoscale three-dimensional spatial-resolved electron spectroscopy for chemical analysis

Rev. Sci. Instrum. 82, 113701 (2011); 10.1063/1.3657156

Ultrafast time-resolved electron diffraction with megavolt electron beams

Appl. Phys. Lett. 89, 184109 (2006); 10.1063/1.2372697

Scanning electron microscope with polarization analysis: Micromagnetic structures in ultrathin films J. Vac. Sci. Technol. B 20, 2535 (2002); 10.1116/1.1519863

Implementation of scanning electron microscopy with polarization analysis using high-efficiency retardingpotential Mott polarimeters

Rev. Sci. Instrum. 70, 246 (1999); 10.1063/1.1149512

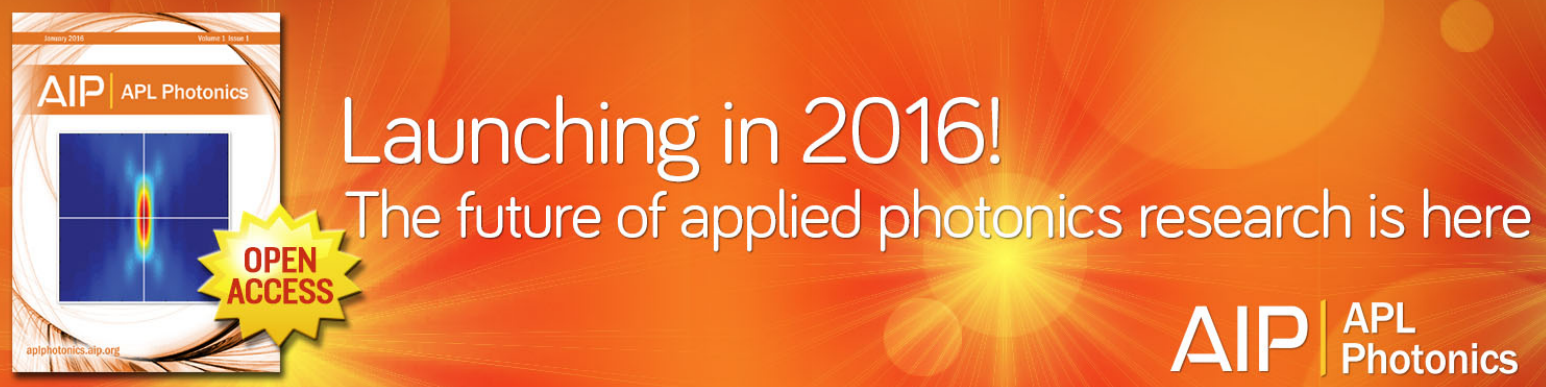




\title{
Time-resolved scanning electron microscopy with polarization analysis
}

\author{
Robert Frömter, ${ }^{1,2, a)}$ Fabian Kloodt, ${ }^{1}$ Stefan Rößler, ${ }^{1}$ Axel Frauen, ${ }^{1}$ Philipp Staeck, ${ }^{1}$ \\ Demetrio R. Cavicchia, ${ }^{1, b)}$ Lars Bocklage, ${ }^{3,2}$ Volker Röbisch, ${ }^{4}$ Eckhard Quandt, ${ }^{4}$ \\ and Hans Peter Oepen ${ }^{1,2}$ \\ ${ }^{1}$ Institut für Nanostruktur-und Festkörperphysik, Universität Hamburg, Jungiusstraße 11, 20355 Hamburg, \\ Germany \\ ${ }^{2}$ The Hamburg Centre for Ultrafast Imaging, Luruper Chaussee 149, 22761 Hamburg, Germany \\ ${ }^{3}$ Deutsches Elektronen-Synchrotron DESY, Notkestraße 85, 22607 Hamburg, Germany \\ ${ }^{4}$ Institute for Materials Science, Christian-Albrechts-Universität zu Kiel, 24143 Kiel, Germany
}

(Received 24 January 2016; accepted 17 March 2016; published online 4 April 2016)

\begin{abstract}
We demonstrate the feasibility of investigating periodically driven magnetization dynamics in a scanning electron microscope with polarization analysis based on spin-polarized low-energy electron diffraction. With the present setup, analyzing the time structure of the scattering events, we obtain a temporal resolution of $700 \mathrm{ps}$, which is demonstrated by means of imaging the field-driven $100 \mathrm{MHz}$ gyration of the vortex in a soft-magnetic FeCoSiB square. Owing to the efficient intrinsic timing scheme, high-quality movies, giving two components of the magnetization simultaneously, can be recorded on the time scale of hours. (C) 2016 AIP Publishing LLC.
\end{abstract}

[http://dx.doi.org/10.1063/1.4945053]

Motivated by the need for data storage devices with ever-faster switching times and higher densities, considerable effort has been spent over the past years to develop techniques that can image magnetic processes at high spatial and temporal resolution. Laser-based methods can routinely access the fs regime, but with $\mu \mathrm{m}$ limited resolution, for instance in scanning Kerr microscopy. ${ }^{1}$ Only with the advent of shorter-wavelength, time-structured synchrotron radiation the required $\mathrm{nm}$ length scale became accessible, e.g., in scanning transmission x-ray microscopy. ${ }^{2}$ At free-electron lasers, recently the combination of $10 \mathrm{fs}$ and $50 \mathrm{~nm}$ imaging was demonstrated using holography. ${ }^{3}$ Electron-based techniques like $\mathrm{x}$ ray photoelectron emission microscopy have been employed at the synchrotron, ${ }^{4}$ and time-resolved magnetic Lorenzmicroscopy is under development. ${ }^{5}$ On much smaller, atomic length scales the development of time-resolved spin-polarized scanning tunneling microscopy has started. ${ }^{6}$

Scanning electron microscopy with polarization analysis (SEMPA), which has been pioneered 30 years ago, ${ }^{7-9}$ is the integration of secondary-electron spin-polarization analysis into an SEM operated in ultrahigh vacuum (UHV). It offers the unique combination of high surface sensitivity (less than $1 \mathrm{~nm}$ in depth) and vectorial magnetic imaging at a lateral resolution down to $3 \mathrm{~nm} .{ }^{10}$ Vectorial imaging means that due to the scattering process used for spin analysis, two components of the local magnetization are detected simultaneously. These can be either both in plane, like in the experiment shown here, or one in plane and the perpendicular component, which can be achieved either by a $90^{\circ}$-bent detector axis ${ }^{9}$ or by controlled spin rotation. ${ }^{11}$ Therefore, the local angle of magnetization can be determined with high accuracy_better than $4^{\circ}$ in the examples of Refs. 12 and 13.

\footnotetext{
a) Author to whom correspondence should be addressed. Electronic mail: rfroemte@physik.uni-hamburg.de.

${ }^{b}$ Present address: GLP Intellectual Property Office, Viale Europa Unita 171, 33100 Udine, Italy.
}

We show in this letter, how imaging on the ns time scale can be efficiently performed in SEMPA, mainly by ex-situ modifications of the signal-processing scheme. At the heart of this scheme is the technical implementation of the spin detector in counting mode, i.e., the asymmetries representing the magnetic contrast are calculated from the electron counts in each of four channels during a fixed time interval. In our case, the detector is of the $\mathrm{W}(001)$ spin-polarized low-energy electron diffraction (SPLEED) type, so the four counters represent the intensities of the four LEED [2,0] beams at $104.5 \mathrm{eV} .{ }^{14}$ Each single electron being diffracted into one of the four beams is amplified by a multichannel-plate assembly into a current pulse of about $2 \mathrm{~ns}$ width. So far, only the number of electrons has been counted to measure the currents of about $170 \mathrm{fA}$ in each LEED beam (corresponding to $10^{6}$ counts/s) with minimum (Poisson) noise. With present-day electronics, it becomes feasible not only to count the number of pulses but also to record the arrival time of each of these pulses in real time with sub-ns resolution. When a periodically driven magnetic state is imaged, the counts can thus be grouped into time slices according to their phase with respect to the driving signal. If we assume equal transit times for all electrons passing through the detector, the time evolution at the detector will differ from the one at the sample surface only by a constant phase. As a result, during a single scan of the image, not only the time-averaged polarization at each pixel is determined but also we obtain a movie of the time evolution of the magnetic sample over the driving period with the selected number of time slices as frames.

The SEMPA instrument used for the time-resolved experiments is described in detail in Ref. 14. We have modified the sample stage to include $\mathrm{GHz}$ wiring and connection of the sample. A pattern generator and an oscilloscope can be attached to generate the ac drive current and monitor its transmission through the sample. During the measurements, the inverted output of the pattern generator is connected to the return line to reduce the voltage modulation at the 
sample. A schematic of the setup is shown in Fig. 1. The single electrons diffracted into the four LEED beams are first amplified in vacuum by a multichannel-plate assembly. Following ex-situ capacitive high-voltage decoupling, the voltage pulses are electronically amplified to give pulses in the $10 \mathrm{mV}-200 \mathrm{mV}$ range with a width of $2 \mathrm{~ns}$. The timing of these pulses is analyzed using a 6 channel multiple-event time-to-digital converter (TDC) running with a base clock of $10 \mathrm{GHz}$ (MCS6A from FAST ComTec $\mathrm{GmbH}$ ). For each cycle, the pattern generator driving the magnetization dynamics sends a start trigger to the TDC. The arrival time of each of the single electron pulses is measured with respect to this start trigger with a time resolution of 100 ps. Pulse detection is performed with respect to a fixed level and leading-edge or constant-fraction timing is used. The timestamps from all pulses together with the channel information are transferred in real time from the TDC to the computer, which then sorts the events into the corresponding time slices and pixels of the image. Thus, the whole time evolution is recorded one pixel after the other. To increase the duty cycle of the measurement, the internal clocks of pattern generator and TDC are synchronized by an additional $10 \mathrm{MHz}$ line, which allows acquiring a large number of cycles at once without waiting for each start trigger.

As test system, the gyrotropic motion of a vortex core in a soft-magnetic microstructure excited by an alternating magnetic field was chosen. Since its first experimental observation in $1984^{15}$ the vortex gyration has been extensively studied, both theoretically ${ }^{16,17}$ as well as experimentally. ${ }^{18-20}$ The sample investigated here consists of a series of $\mathrm{FeCoSiB}$ squares with a side length of $3.5 \mu \mathrm{m}$, structured from $60 \mathrm{~nm}$ thick $\mathrm{Fe}_{70} \mathrm{Co}_{8} \mathrm{Si}_{12} \mathrm{~B}_{10}$ on top of a gold microstrip on sapphire (see Fig. 2). FeCoSiB, while having a similarly low magnetic anisotropy as Permalloy, shows a much stronger image contrast in SEMPA due to the higher spin polarization of iron compared to nickel. For the microstrip, $150 \mathrm{~nm}$ gold where sputtered onto a $24 \mathrm{~nm}$ chromium seed layer on polished $\mathrm{Al}_{2} \mathrm{O}_{3}(0001)$.

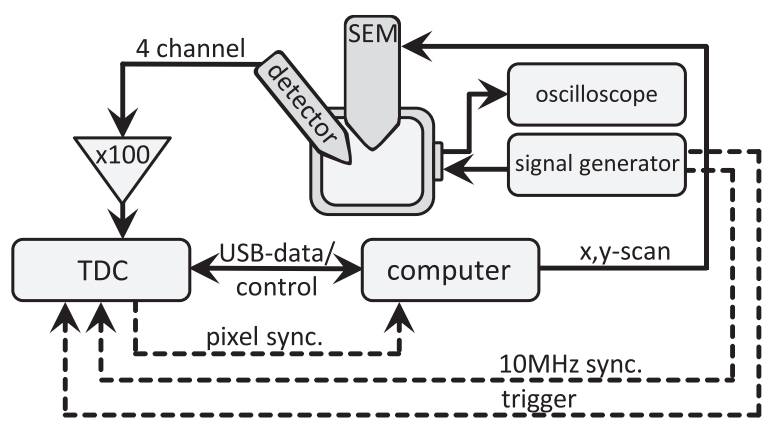

FIG. 1. Sketch of the wiring of the time-resolved SEMPA. The SEM column, spin-detector, and UHV chamber are schematically drawn in the center. A signal generator is connected via SubMiniature-A-feedthroughs to the sample holder to drive the current for the periodic magnetic excitation. The return current from the microstrip can be monitored via a scope. During experiments, an inverted driving voltage from the signal generator is applied to the return lead. For synchronization, the TDC receives a trigger signal at the beginning of each cycle. An additional $10 \mathrm{MHz}$ signal synchronizes the internal clock of both devices. The TDC creates a timestamp in multiples of $100 \mathrm{ps}$ for each count on any of the four detector channels, specifying the channel and delay with respect to the trigger. This data is then sorted and counted into time slices by a computer. After data transfer, the computer moves the SEM beam to the next position.

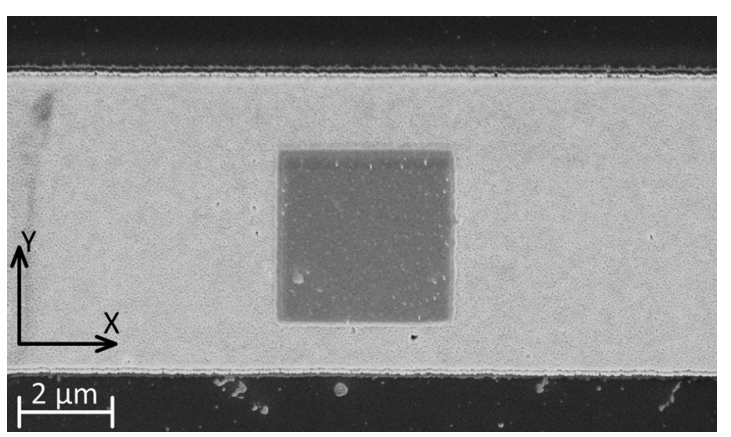

FIG. 2. SEM image of the $60 \mathrm{~nm} \mathrm{Fe}_{70} \mathrm{Co}_{8} \mathrm{Si}_{12} \mathrm{~B}_{10}$ sample. A $3.5 \mu \mathrm{m}$ sized square is structured in the magnetic film, that has been sputtered on a microstrip prepared from $\mathrm{Au}(150 \mathrm{~nm}) / \mathrm{Cr}(24 \mathrm{~nm})$ on a polished sapphire $(0001)$ substrate. According to the indicated coordinate system, a current flowing in positive $\mathrm{x}$-direction will generate an Oersted field in the negative y-direction at and above the position of the square.

The $5 \mu \mathrm{m}$ wide microstrip was then structured using positive photoresist and subsequent low-energy argon milling. Following an $\mathrm{O}_{2}$-plasma cleaning, the $60 \mathrm{~nm} \mathrm{FeCoSiB} \mathrm{film}$ and a $3 \mathrm{~nm}$ tantalum cap layer were sputtered onto the structure at room temperature. ${ }^{21}$ Using e-beam lithography with a negative resist the squares were patterned and finally structured by low-energy argon milling. The tantalum cover was removed inside the SEMPA chamber by argon ion milling before the experiment.

Static SEMPA measurements at remanence show that all squares are in the magnetic Landau state. An alternating current is driven through the gold microstrip to create an Oersted field at the position of the $\mathrm{FeCoSiB}$ squares. This field is oriented perpendicular to the wire and primarily within the film plane. Far below resonance, this field increases (decreases) the size of the Landau domain that is aligned (anti)parallel with the field to minimize the Zeeman energy, which results in a linear oscillation of the vortex along the wire axis. The driving frequency of $100 \mathrm{MHz}$ used in this experiment is well below the resonance, which is expected around $250 \mathrm{MHz}$ for the prepared $\mathrm{FeCoSiB}$ squares. To obtain dynamics with reasonable amplitude, we therefore use a current of $48 \mathrm{~mA}$ amplitude, driven by a potential difference of $2.4 \mathrm{~V}_{\mathrm{PP}}$ along the wire. This voltage drop and current affect the primary electron beam. While the Oersted field of the current results in an x-deflection of the beam, the electric potential causes a $y$-deflection. The reason for the latter motion is that the primary electrons are permanently deflected in $-\mathrm{y}$ direction by the static electric field from the first electrode of the spin detector. The magnitude of the resulting shift depends on their kinetic energy, which is modulated by the sample potential. To reduce this effect, we applied an antisymmetric drive voltage of $\pm 1.2 \mathrm{~V}_{\mathrm{PP}}$ to both sides of the microstrip, so that in the center of the strip the potential remained constant. This also maintains constant and optimized working conditions for the spin analyzer, as a potential modulation at the sample modulates the kinetic energy of the secondaries. In Fig. 3(c), the image shift due to the deflection of the primary beam is plotted, as extracted from the apparent motion of the $\mathrm{FeCoSiB}$ square in the sum image, i.e., the sum of the four spin images. Using error functions to fit the edges, a sinusoidal shift is obtained. We find peak-to-peak amplitudes of $70 \mathrm{~nm}$ in $\mathrm{x}$ - and $60 \mathrm{~nm}$ in $\mathrm{y}$-directions, corresponding to about 2 pixels in the 

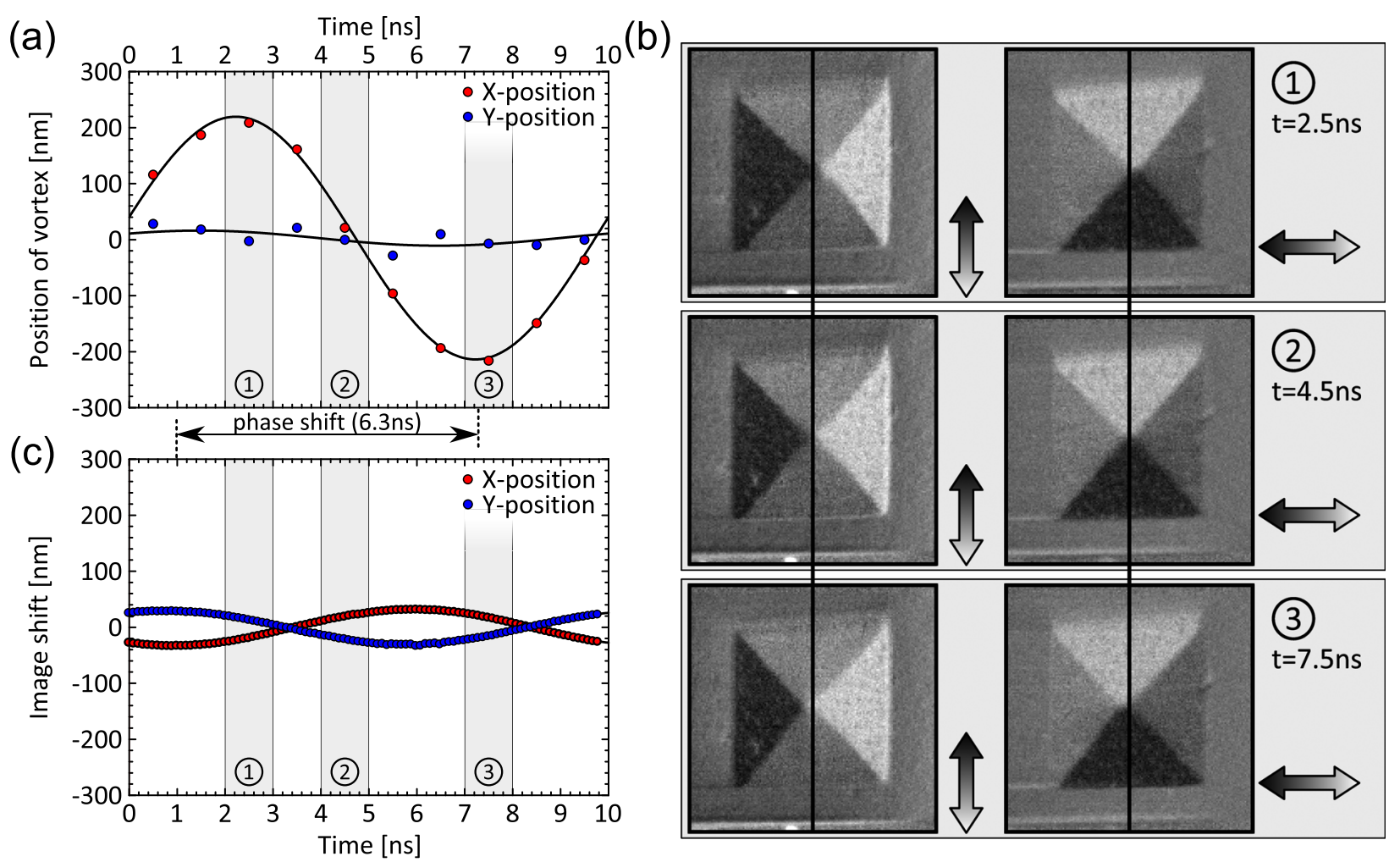

FIG. 3. Time-resolved SEMPA measurement of the vortex gyration. An antisymmetric drive voltage of $\pm 1.2 \mathrm{~V}_{\mathrm{PP}}(100 \mathrm{MHz})$ has been applied to both ends of the microstrip. Panel (b) shows three image pairs from a movie containing 10 frames of $1 \mathrm{~ns}$ each at the given delays. The image contrast corresponds to the inplane magnetization along the two directions indicated by arrows and reveals the Landau state. A current-driven motion of the vortex structure along the $\mathrm{x}$-direction (horizontal) is clearly visible. The linked multimedia file shows the two component images together with the sum image assembled to a movie containing all 10 frames. To quantify this observation the position of the vortex in $\mathrm{x}$ and $\mathrm{y}$ directions is extracted. The displacement as a function of delay time is plotted in (a). The displacements as well as the images in (b) are already corrected for the small image shift given in (c), which has been obtained from the apparent motion of the whole structure in the sum image. (Multimedia view) [URL: http://dx.doi.org/10.1063/1.4945053.1]

image. This systematic and easy-to-determine image shift is already corrected in the following magnetic images and in the analysis of the vortex motion.

Fig. 3(b) (Multimedia view) shows the images taken with the two polarization axes sensitive to the two in-plane components of magnetization for three different delay times $(2.5 \mathrm{~ns}$, $4.5 \mathrm{~ns}$, and $7.5 \mathrm{~ns}$ ). Black/white arrows indicate the magnetization component in the respective image. A primary beam of $6 \mathrm{nA}$ at $7 \mathrm{kV}$ was used. The raster of $150 \times 150$ pixels was scanned 300 times, using automatic drift correction between frames. We measured at the full time resolution of the TDC (100 ps) for a total dwell time of $252 \mathrm{~ms}$ per pixel, corresponding to $94 \mathrm{~min}$ active measurement time. With the present software, the total measurement took 266 min due to internal dead times of the TDC and processing delays of the computer. The development of more efficient device communication and timings will further increase the measurement efficiency. The contrast in the magnetic component images is much smaller than in the sum image, where the full time resolution was used in the analysis. To improve the contrast-to-noise ratio (CNR), the counts of 10 contiguous time slices have been summed up during data processing and appointed to a single slice of $1 \mathrm{~ns}$. A displacement of the vortex is clearly visible in the images. To quantify the displacement, the time-dependent vortex position in $\mathrm{x}$ and $\mathrm{y}$ directions is analyzed in Fig. 3(a). A sinusoidal motion, predominantly along the wire axis, is found with peak-to-peak amplitude of $440 \mathrm{~nm}$. This motion is consistent with the expected excitation of the structure driven below the resonance frequency. The amplitude of vortex motion is much larger than the previously discussed image shift. A phase difference between image shift in x-direction (Oersted field) and vortex displacement of $6.3 \mathrm{~ns}$ can be determined from comparing the positions of the minima in both graphs. In this way, not only the amplitude of a driven dynamics but also the phase with respect to a driving current or field can be extracted and analyzed.

To determine the time resolution of our instrument, we analyze the motion blur of a sharp sample edge as a result of the image shift. Here, we applied a symmetric drive voltage to obtain a large image shift in y direction. Fig. 4 shows the width and position of the edge of a square (see inset) as a function of time during the driving cycle. To obtain a stronger effect, a square wave of $110 \mathrm{MHz}$ was used instead of a harmonic excitation. The shape of the driving voltage coincides very well with the observed image shift, so no direct bandwidth limiting effect is apparent from the edge position. During the abrupt voltage change, however, a widening of the edge is observed, which is due to the temporal overlap of images from different real times. This motion blur effect is proportional to the velocity of the moving image $v(t)$. In analogy to spatial resolution, we define the temporal resolution as $2 \sigma_{t}$ (approximately the full width at half maximum) of a Gaussian convolution or blur in time, where $\sigma$ denotes the standard deviation. The observed edge width $2 \sigma(t)$ is the Pythagorean sum of static edge width $2 \sigma_{0}$ and motion blur $2 \sigma_{t} v(t)$. The dashed line in Fig. 4 indicates a peak velocity of 


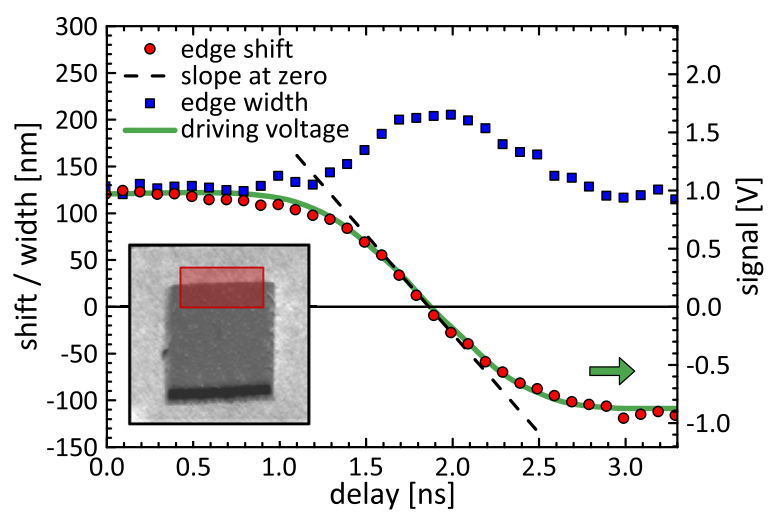

FIG. 4. Determining the time resolution. Position (red dots) and width (blue squares) of the upper edge of the square (see inset) for a symmetric squarewave driving voltage at $110 \mathrm{MHz}$ (green line; right scale). The image shift closely follows the driving voltage, which indicates a time resolution better than the bandwidth of the signal generator. However, the spatial image resolution given by the blue squares is reduced (the given $2 \sigma$ value of a fitted Gaussian blur increases by $70 \mathrm{~nm}$ ) where the velocity of the image shift is maximum. The size of this motion blur effect allows extracting the time resolution in our setup using the peak velocity of $(210 \pm 10) \mathrm{m} / \mathrm{s}$ from the slope of the edge shift. As a result, we find a time resolution of $(720 \pm 80)$ ps.

$(210 \pm 10) \mathrm{m} / \mathrm{s}$ of the edge shift, while the edge width varies from $2 \sigma_{0}=(130 \pm 10) \mathrm{nm}$ to $(200 \pm 10) \mathrm{nm}$ at peak velocity. This results in a time resolution of $2 \sigma_{t}=(0.72 \pm 0.08) \mathrm{ns}$.

Two factors potentially limit the time resolution in the present setup. One is the uncertainty in timing of the pulses that is caused by the wide pulse height distribution from a Chevron channelplate assembly ${ }^{22}$ considering the pulse width of $2 \mathrm{~ns}$. We observed that using constant-fraction instead of leading-edge timing improves the time resolution by about 250 ps. As Chevron channelplate assemblies with 150 ps pulse width and $20 \mathrm{GHz}$ TDC are commercially available, the limit from the detection side will be below 100 ps at present.

The second limitation is given by time jitter of the electron transit through the spin analyzer. As the secondary electrons emitted from the sample possess an energy spread of some $\mathrm{eV}$ and a cosine-type angular distribution, electrons emitted at the same time at the sample will hit the detector at slightly different times. Trajectory calculations show a total transit time of about $11 \mathrm{~ns}$ for our detector, during which a jitter of about $0.6 \mathrm{~ns}$ is picked up. Approximately $20 \%$ of this jitter is accumulated during initial acceleration of the secondary electrons into the spin detector, another $40 \%$ during transit at scattering energy $(104.5 \mathrm{eV})$, and the final $40 \%$ at the retarding grid, blocking the additional secondary electrons that originate from the tungsten surface. It can be expected that with an optimized detector design a factor two to four in time resolution could be gained.

The acquisition time in SEMPA depends on the required CNR of an image, where the noise is given by the Poisson statistics of the number of single electron counts in each pixel. ${ }^{14}$ For clean iron or cobalt, with an average secondaryelectron spin polarization of about $40 \%$, a good CNR of 25 can be obtained at a dwell time of $10 \mathrm{~ms}$ per pixel and $6 \mathrm{nA}$ primary current for our detector. Thus, a reasonably detailed image containing $250 \times 250$ pixels will take about $10 \mathrm{~min}$. The data acquisition scheme for time-resolved SEMPA presented here has the advantage that the total acquisition time for a movie scales only linearly with number of frames. This can be easily understood from the fact that the TDC is running at a fixed duty cycle $(30 \%-90 \%$ depending on communication overhead), so a fixed fraction of counts occurring during the measurement is constantly filled into one of the frames. In contrast, for a stroboscopic scheme, where the beam or detector was blanked for all times, but for the currently measured frame, an unfavorable scaling with the square of the number of frames resulted. A peculiar consequence of this linear scaling is that the acquisition time is independent of the actual duration of the frames and thus the time scale. To record 10 frames of $1 \mathrm{~ns}$, each requires the same acquisition time as to record 10 frames of $1 \mathrm{~ms}$. However, a process where the initial dynamics needs to be analyzed on the $1 \mathrm{~ns}$ scale while the restoration period needs $1 \mathrm{~ms}$ required 100 times longer to record. It should also be noted that for the gyrating vortex presented above, on average for every 15 rotations of the vortex an electron is actually counted in one of the four channels.

Time-resolved SEMPA is introduced as a powerful tool for the study of nanometer-scale cyclic magnetization dynamics. It covers the whole range from nanosecond timescales down to the static case. In addition to the time evolution of a magnetic system, the relative phase of the driving current can be extracted from the image shift. The unique properties of SEMPA, i.e., surface sensitivity and vectorial imaging, as well as the benefits of working on a solid surface with the potential for temperature control and single-crystal epitaxy are maintained.

The development was supported by DFG within SFB668.

${ }^{1}$ W. K. Hiebert, A. Stankiewicz, and M. R. Freeman, Phys. Rev. Lett. 79, 1134 (1997).

${ }^{2}$ Y. Acremann, V. Chembrolu, J. P. Strachan, T. Tyliszczak, and J. Stöhr, Rev. Sci. Instrum. 78, 014702 (2007).

${ }^{3}$ T. Wang, D. Zhu, B. Wu, C. Graves, S. Schaffert, T. Rander, L. Müller, B. Vodungbo, C. Baumier, D. P. Bernstein et al., Phys. Rev. Lett. 108, 267403 (2012).

${ }^{4}$ A. Krasyuk, A. Oelsner, S. A. Nepijko, A. Kuksov, C. M. Schneider, and G. Schönhense, Appl. Phys. A 76, 863 (2003).

${ }^{5}$ H. S. Park, J. S. Baskin, and A. H. Zewail, Nano Lett. 10, 3796 (2010).

${ }^{6}$ S. Loth, M. Etzkorn, C. P. Lutz, D. M. Eigler, and A. J. Heinrich, Science 329, 1628 (2010).

${ }^{7}$ K. Koike and K. Hayakawa, Jpn. J. Appl. Phys. 23(3), L187 (1984).

${ }^{8}$ J. Unguris, D. T. Pierce, and R. J. Celotta, Rev. Sci. Instrum. 57, 1314 (1986).

${ }^{9}$ H. P. Oepen and J. Kirschner, Scanning Microsc. 5, 1 (1991); J. Phys. 49, 1853 (1988).

${ }^{10}$ K. Koike, Microscopy 62, 177 (2013).

${ }^{11}$ T. Kohashi, M. Konoto, and K. Koike, Rev. Sci. Instrum. 75, 2003 (2004).

${ }^{12}$ S. Hankemeier, R. Frömter, N. Mikuszeit, D. Stickler, H. Stillrich, S. Pütter, E. Y. Vedmedenko, and H. P. Oepen, Phys. Rev. Lett. 103, 147204 (2009).

${ }^{13}$ M. Seifert, L. Schultz, R. Schäfer, V. Neu, S. Hankemeier, S. Rössler, R. Frömter, and H. P. Oepen, New J. Phys. 15, 013019 (2013).

${ }^{14}$ R. Frömter, S. Hankemeier, H. P. Oepen, and J. Kirschner, Rev. Sci. Instrum. 82, 033704 (2011).

${ }^{15}$ B. E. Argyle, E. Terrenzio, and J. C. Slonczewski, Phys. Rev. Lett. 53, 190 (1984).

${ }^{16}$ K. Y. Guslienko, B. A. Ivanov, V. Novosad, Y. Otani, H. Shima, and K. Fukamichi, J. Appl. Phys. 91, 8037 (2002).

${ }^{17}$ B. Krüger, A. Drews, M. Bolte, U. Merkt, D. Pfannkuche, and G. Meier, Phys. Rev. B 76, 224426 (2007). 
${ }^{18}$ A. Puzic, B. Van Waeyenberge, K. W. Chou, P. Fischer, H. Stoll, G. Schutz, T. Tyliszczak, K. Rott, H. Bruckl, G. Reiss, I. Neudecker, T. Haug, M. Buess, and C. H. Back, J. Appl. Phys. 97, 10 E704 (2005).

${ }^{19}$ J.-S. Kim, O. Boulle, S. Verstoep, L. Heyne, J. Rhensius, M. Kläui, L. J. Heyderman, F. Kronast, R. Mattheis, C. Ulysse, and G. Faini, Phys. Rev. B 82, 104427 (2010).
${ }^{20}$ T. Kamionka, M. Martens, A. Drews, B. Krüger, O. Albrecht, and G. Meier, Phys. Rev. B 83, 224424 (2011).

${ }^{21}$ V. Hrkac, E. Lage, G. Köppel, J. Strobel, J. McCord, E. Quandt, D. Meyners, and L. Kienle, J. Appl. Phys. 116, 134302 (2014).

${ }^{22}$ J. L. Wiza, Nucl. Instrum. Methods 162, 587 (1979). 